Hand, dass unter Umstănden diese letzteren durch ibren Sitz gefährlicher wie jene werden könuen.

Zwischen diesen Extremen liegen als Uebergänge diejenigen Formen, wie Sarcome, Myxome, welche von den verschiedenen Beobachtern bald mehr zur einen, bald zur anderen Grenze der Scala gerechnet werden.

4.

\title{
Spontane Gangrän am Fusse, Amputation des Oberschenkels, Heilung.
}

\author{
Von Dr. B urow jun. in Königsberg.
}

Abraham Borker, 40 Jahre alt, jüdischer Lehrer aus Kowno, von schwächlicher Constitution, aber sonst gesund, bekam, nachdem er einige Wochen vorher an "rheumatischen" Schmerzen des rechten Fusses gelitten hatte, Mitte Mai 1866 obne veranlassende Ursache Brand der letzten Phalanx der rechten grossen Zehe.

Die dortigen Aerzte exarticulirten nach 3 Monaten im ersten Gelenk; als aber die Gangrän weiter vorschritt, amputirten sie dicht dahinter in der Mitte der 2ten Phalanx.

Patient stellte sich mir Anfangs September hier vor. Es war jetzt der Rest der Zehe schwarz, eine seröse Jauche aussondernd, während aus der übelriechenden Wundläche der schwarze nekrotische Phalanzknochen mit rauher Sägefläche hervorragte. Der ganze Fuss war dabei kälter, als der gesunde, etwas geröthet und Ieicht ödematös. Beweglichkeit der übrigen Zehen war vorhanden. Während die Arteria poplitea der gesunden Seite, wenn auch undeutlich, unter dem zufüblenden Finger pulsirte, so war in der rechten Kniekehle statt ihrer nur ein solider Strang von der Dicke eines Fingers bemerkbar, an dem ich keine Spur von Putsation entdecken konnte. Die Cruralis erwies sich an beiden Schenkeln intact. Unterhalb des Kniees war am kranken Gliede keine Arterienpulsation zu entdecken. Patient wurde ausser dem starken Gestank hauptsächlich durch fortwährende Schmerzen gepeinigt, welche ihm völlig den Schlaf raubten. Das Herz erwies sich als gesund.

Ich entschloss mich, da sich scheinbar eine Demarcationslinie gebildet hatte, zur operativen Entfernung, und machte als nächste noch im Gesunden ausführbare Operation die Resection des Capitul. oss. metatars. primi, welches ich vermittelst der Kettensäge am 10. September 1866 extfernte, wobei ich 2 Lappen aus, wie es schien, intacten Weichtheilen bildete und bei sehr geringer Blutung keiner Unterbindung bedurfte: Allein schon am 2ten Tage zeigten sich unter Schüttelfrösten die ersten Spuren des von Neuem auftretenden Brandes, durch Missfarbigwerden der Lappen. Der Prozess schritt jetzt langsam, aber stetig vor, und ergriff im Laufe der nächsten 3 Wochen die anderen Zehen, welche erst empfindangslos, dann livid, blau, schliesslich schwarz wurden. Der Gebrauch des Chinadecocts mit

Archiv f. pathol. Anat. Bd. XXXVIII. Hft. 4. 
Säuren, verbunden mit Umschlägen von essigsaurer Thonerde und später Campferwein nützte nichts; der Patient kam mehr und mehr herunter, der Brand schritt auf den Fuss vor. Derngemäss entschloss ich mich zur Absetzung des kranken Gliedes und wählte ans gleich zu nennenden Gründen die Amputation des Oberschenkels im unteren Drittheil. Ich führte die Operation am 4.0ctoher, also circa 5 Monate nach Auftreten der krankheit, aus, und bildete 2 beinahe gleichgrosse Lappen, einen äusseren und einen inneren. Es zeigte sich, dass grade an der Operationsstelle die Behinderung in der Arterie lag. Denn ich fand keine eigentliche Femoralarterie, welche doch normaler Weise bier noch ein beträchtliches Lumen haben soll. Vielmehr waren statt ihrer mehrere kleine, wenig spritzende Arterien vorhanden, welche durch Bindegewebe zusammengekittet, einen fingerdicken Strang ausmachten, und einzeln unterbunden werden mussten. Dafür war überall eine bedeutende Entwickelung von Collateralgefässen zu constatiren, so dass mehr Unterbindungen, als gewöhnlich, nöthig wurden. Eine beträchtliche Knochenblutung stellte sich ein, welche auf directe Compression mittelst Charpiebausch gegen die offene Markhöhle (ein sonst probates Mittel) nicht stand, zu deren Stillung vielmehr die Application des Ferrum candens erforderlich wurde. Ja selbst die kleinen am Nerv. ischiadicus verlaufenden Gefässe waren so stark entwickelt, dass der frei zu Tage liegende Nerv zwischen den Fingern eine deutliche Pulsation zeigte, und ich zwei spritzende Gefässe an ihm unterbinden musste. Der Heilungsverlauf war ein sehr günstiger. Reaction war nur in den ersten Tagen rorhanden, Patient sass bereits nach 10 Tagen im Bett; nach 3 Wochen war fast Alles verheilt, nach 1 Monat konnte er aus der Anstalt in sein Privatlogis entlassen werden, und hat der Stumpf ein sehr schönes Polster.

Die Section des Gliedes zeigte, dass die Arterienverengerung von der Amputationsstelle nach unten weiter ging, während die Tibialis postic. sich zwar wegsam aber ebenfalls ein bedeutend verengtertes Lumen darbot. Von Atheromatose oder Embolien konnte ich nichts naclwweisen, vielmehr scheint die Hemmung und Schwächung des Blutstroms durch die Verengerung der Arterien die eigentliche Ursache der Gangrän gewesen za sein.

Ich habe mir erlaubt, diesen Fall bier zu beschreiben, weil die Ansichten der Autoren über die Zweckmässigkeit der Amputation bei Altersbrand noch so sebr divergiren, und grade die Zusammenstellung solcher Fälle, speciell, wenn sie verschieden behandelt wurden, dem Arzt die Richtschnur seines Handelns an die Hand geben können.

Es ist unter solchen Umständen die Frage zu entscheiden, ob man ruhig der Natur die Abstossung des Kranken überlassen solle, oder ob dieser langsame Prozess durch chirurgische Hälfe abzukürzen sei.

In meinem Falle war einerseits bereits der 3te operative Eingriff ausgeführt, ohne dass es möglich gewesen wäre; die Gangrän aufzubaiten, und es Lonnte jetzt nur noch von einer bedeutenden Operation die Rede sein, so dass es fraglich wurde, ob der ohnehin geschwächte Körperzustand des Patienten einen solchen Insult ertragen würde. Auf der anderen Seite waren alle bisherigen Absetzungen zu nahe den kranken Theilen gemacht worden, und es stand bei der Langsamkeit des Vorschreitens eine so grosse Störung der Gesundheit durch Schlaflosigkeit, 
Schmerzen und Jaucheresorption zu befürchten, dass eher das Leben erlöschen, als die Demarcation vollendet sein würde.

Vergleichen wir aber die Erfolge, wie sie z. B. noch in letzter Zeit Jäsche *) zusammengestellt hat, so werden wir uns unter Berücksichtigung der übrigen Erscheinungen, oft zur Amputation entschliessen können.

Wenn ein Arterienverschluss oder eine Verengerung vorlag, so kam es wesentlich darauf an, zu entscheiden, wo die unwegsame Stelle lag, und ferner, wo zu operiren war. Die Entscheidung der ersten Frage war nicht leicht.

Im oberen Dritttheil des Oberschenkels pulsirte die Arterie ganz deutlich, in der Kniekehle dagegen glaubte ich einen soliden Strang statt ibrer zu fählen, ebenso war unterhalb keine Pulsation nachweisbar. Lag nun der Verschluss resp. die Verengerung grade hier, oder war die Pulsation der Poplifea aus anderen Gründen, z. B. des stärkeren Fettpolsters wegen, nicht fühlbar und der Strang irgend ein anderes 0rgan? Und ferner war es mehr als fraglich, ob sich der Collateralkreislauf bereits so weit ausgebildet hahen würde, dass das obere Dritttbeil des Unterschenkels gut versorgt würde, so dass bereits bier die Absetzung möglich sei. Denn von einer Amputation im Fuss konnte kaum die Rede sein, weil hier die Theile bereits krank waren, die Wahrscheinlichkeit' also des Wiederauffammens des Brandes am Stumpfe zu gross sein musste. Oder aber, wenn ich möglichst vom Kranken entfernt operiren wollte, so war der Zweifel berechtigt, ob die vermehrte Gefahr einer Oberschenkelamputation durch die grössere Sicherheit normaler Gefässlumina aufgewogen werden würde.

Ich erlaube mir hier an einen von Dardel**) aus der Lücke'schen Klinik in Bern mitgetheilten Fall zu erinnern, wo unter ahnlichen Verhältnissen 6 Monate nach Auftreten der Gangrän an der Zehe die Pirogoff'sche Operation 1-2 Zoll oberhalb der kranken Gewebe gemacht wurde, wo dann aber das Leben des Patienten durch Weiterschreiten des Brandes 3 Zoll über die Operationsstelle und durch pyämische Erscheinungen in die grösste Gefahr kam, und wo endlich 3 Wochen nach der ersten Abtragung die vorstehenden Enden der Knochen und des Nervus tibial. entfernt werden mussten. Dass ich eine von dem eben citirten Falle abweichende Methode befolgte, geht aus dem Obigen hervor; der Erfolg rechtfertigte mich, indem die Gangrän nicht weiter auftrat, wenn auch zugestanden werden muss, dass die Verstünmelung eine bedeutende war.

Charakteristisch für diese Form des Brandes scheinen mir 1) die sehr heftigen und andauernden Schmerzen, welche selbst durch subeutane Morphium-Injectionen nur momentan betäubt wurden; 2) das äusserst langsame aber unaufhörliche Vorschreiten, indem mehr als 2 Wochen erforderlich waren, ehe eine Zehe von den ersten Zeichen des Brandes ab, völlig gangränescirt war; und 3) die mangelnde Begrenzung in die Tiefe, so dass die nekrotischen Gewebstheile nicht, wie es sonst wohl geschieht, sich einfach abheben liessen, sondern nur durch Scheerenschnitte entfernt werden konnten.

*) Langenbeck, Archiv VI., 3, S. 694.

**) Berlin. Klinisch. Wochenschr. No. 52, 1866. 